\title{
Explicit and Implicit Methods for Fractional Diffusion Equations with the Riesz Fractional Derivative
}

\author{
Ahmad Reza Haghighi ${ }^{1 *}$, Hamideh Hoseini Ghejlo' and Nasim Asghari
}

'Department of Mathematics, Urmia University of Technology, Urmia, Iran; ah.haghighi@gmail.com,h.hoseini5761@gmail.com

²Department of Mathematics, Islamic Azad University, Central Tehran Branch, Tehran, Iran; nasimsana@yahoo.com

\begin{abstract}
In this paper, a fractional diffusion equation by using the explicit numerical method in a finite domain with second-order accuracy which includes the Riesz fractional derivative approximation is studied. For the Riesz fractional derivative approximation, "fractional centered derivative" approach is used. The error of the Riesz fractional derivative to the fractional centered difference is calculated. We used the implicit numerical method to solve the fractional diffusion equation and also investigated the stability of explicit and implicit methods. The maximum error of the implicit method for fractional diffusion equation with using fractional centered difference approach is shown by using the numerical results.
\end{abstract}

Keywords: Riesz Fractional Derivative Operator, Implicit Method, Explicit Method, Fractional Central Difference.

2010 Mathematics Subject Classifications: 34A08, 35AXX

\section{Introduction}

Fractional differential equations are used frequently in science and engineering, such as: fractional diffusion and wave equations $[1,2]$, electrical systems [3], viscoelasticity theory [3], control systems [3], biomedical engineering [4] finance [5] and the economic analysis of the stock prices. Let the $\frac{\partial^{\alpha}}{\partial|x|^{\alpha}}$ be the Riesz fractional derivative operator for $1<\alpha \leq 2$ that is defined in [6-8] as follows:

$\frac{\partial^{\alpha} u(x, t)}{\partial|x|^{\alpha}}=-\frac{1}{2 \cos \left(\frac{\alpha \pi}{2}\right) \Gamma(2-\alpha)} \frac{d^{2}}{d x^{2}} \int_{-\infty}^{\infty}|x-\xi|^{1-\alpha} u(\xi, t) d \xi$

$$
\Gamma(z)=\int_{0}^{\infty} t^{z-1} e^{-t} d t
$$

${ }^{*}$ Corresponding author:

Ahmad Reza Haghighi (ah.haghighi@gmail.com)
We consider the following equation in a finite domain associated with initial and Dirichlet boundary conditions

$$
\begin{gathered}
\frac{\partial u(x, t)}{\partial t}=D \frac{\partial^{\alpha} u(x, t)}{\partial|x|^{\alpha}}+f(x, t), \quad a<x<b, \quad 0<t \leq T \\
\begin{cases}u(a, t)=\varphi(t), & 0<t \leq T \\
u(b, t)=\Phi(t), & 0<t \leq T \\
u(x, 0)=\psi(x), & a \leq x \leq b\end{cases}
\end{gathered}
$$

where $D>0$ is diffusion coefficient, and $f(x, t), \varphi(t), \Phi(t), \psi(x)$ are sufficiently smooth functions. To estimate the Riesz fractional derivatives, Grunwald-Letnikov derivative approximation, we use scheme of order $\mathrm{O}(\mathrm{h})$ [9-15]. Meerschaert and Tadjeran [11] and Tadjeranetal. [12] applied the Crank-Nicolson method with Grunwald-Letnikov 
derivative approximation to a linear diffusion equation, which has an independent fractional derivative, represented that the used method is unconditionally stable for given problems. Shen and et al. [13] applied implicit and explicit finite difference methods with Grunwald-Letnikov derivative approximation to a linear Riesz fractional diffusion equation, and showed that the explicit method is conditionally and the implicit models is unconditionally stable for given problems. To improve the convergence order of used approximation, they used the Richordsons extrapolation [11-14]. Zhang and Liu [15] applied the implicit finite difference method with Grunwald-Letnikov derivative approximation to a nonlinear Riesz fractional diffusion equation and showed that the used method is stable for small time. As a new approach, Ortigueire [16] defined the "fractional central derivative" and proved that the Riesz fractional derivative of an analytic function can be represented by fractional central derivative.

In Section 2, we show that the fractional central difference is approximated with $O\left(h^{2}\right)$ accurate to the Riesz fractional derivative for $1<\alpha \leq 2$. In Section 3 and 5 we applied the explicit and implicit method for the problem (2) and (3) by using the fractional centered difference discretization. In Section 4 and 6 we give the stability properties of the explicit and implicit method for the problem (2) and (3). Finally, in the last section we presented a numerical solution of an example by using the implicit method.

\section{Approximation by Fractional Centered Difference}

In [16] for $\alpha>-1$ the fractional centered difference is defined by

$$
\Delta_{h}^{\alpha} \theta(x)=\sum_{k=-\infty}^{\infty} \frac{(-1)^{k} \Gamma(\alpha+1)}{\Gamma\left(\frac{\alpha}{2}-k+1\right) \Gamma\left(\frac{\alpha}{2}+k+1\right)} \theta(x-k h)
$$

and it is shown that

$$
\lim _{h \rightarrow 0} \frac{\Delta_{h}^{\alpha} \theta(x)}{h^{\alpha}}=\lim _{h \rightarrow 0} \frac{1}{h^{\alpha}} \sum_{k=-\infty}^{\infty} \frac{(-1)^{k} \Gamma(\alpha+1)}{\Gamma\left(\frac{\alpha}{2}-k+1\right) \Gamma\left(\frac{\alpha}{2}+k+1\right)} \theta(x-k h)
$$

represents the Riesz fractional derivative (1) for the case of $1<\alpha \leq 2$. we use Eq. (5) as a discretization to the Riesz fractional derivative. Therefore, we express the following property and lemma.

\section{Property 2.1.}

Let $g_{k}=\frac{(-1)^{k} \Gamma(\alpha+1)}{\Gamma\left(\frac{\alpha}{2}-k+1\right) \Gamma\left(\frac{\alpha}{2}+k+1\right)}$ be the coefficients of the centered finite difference approximation (5) for $k=0$, $\pm 1, \pm 2, \ldots$, and $\alpha>-1$. Then

$$
g_{k} \geq 0
$$

$g_{k}=g_{-k} \leq 0$ for $|k| \geq 1$

\section{LEMMA 2.2.}

Let $\mathrm{f} \in C^{5}(R)$ and all derivatives up to order five belong to $L_{1}(R)$ and

$$
\Delta_{h}^{\alpha} \mathrm{f}(x)=\sum_{k=-\infty}^{\infty} \frac{(-1)^{k} \Gamma(\alpha+1)}{\Gamma\left(\frac{\alpha}{2}-k+1\right) \Gamma\left(\frac{\alpha}{2}+k+1\right)} f(x-k h)
$$

be the fractional centered difference. Then

$$
-h^{-\alpha} \Delta_{h}^{\alpha} f(x)=\frac{\partial^{\alpha} f(x)}{\partial|x|^{\alpha}}+O\left(h^{2}\right)
$$

When $h \rightarrow 0$ and $\frac{\partial^{\alpha} f(x)}{\partial|x|^{\alpha}}$ is the Riesz fractional derivative for $1<a \leq 2$.

if $\mathrm{f}^{*}$ is defined by

$$
f^{*}(x)=\left\{\begin{array}{cc}
f(x), & x \in[a, b] \\
0, & x \notin[a, b]
\end{array}\right.
$$

such that $f^{*}(x) \in C^{5}(\mathrm{R})$ and all derivatives up to order five belong to $L_{1}(R)$ then from Lemma 2.2, we have

$$
\frac{\partial^{\alpha} f^{*}(x)}{\partial|x|^{\alpha}}=-h^{-\alpha} \sum_{k=-\infty}^{\infty} g_{k} f^{*}(x-k h)+O\left(h^{2}\right)
$$

Since $f^{*}(x)=0$ for $x \notin[a, b]$ we get

$$
\frac{\partial^{\alpha} f(x)}{\partial|x|^{\alpha}}=-h^{-\alpha} \sum_{k=\frac{b-x}{h}}^{\frac{x-a}{h}} g_{k} f(x-k h)+O\left(h^{2}\right)
$$

Where $h=\frac{b-a}{m}$, and $m$ is the number of partitions of the interval $[a, b]$.

\section{The Explicit Discretization for Fractional Diffusion Equation}

Explicit discretization for the equations 2 and 3 is

$$
\frac{u_{i}^{j+1}-u_{i}^{j}}{\tau}=-D h^{-\alpha} \sum_{k=-m+i}^{i} g_{k} u_{i-k}^{j}+f_{i}^{j+\frac{1}{2}}
$$


Or

$$
u_{i}^{j+1}=u_{i}^{j}-\frac{D_{\tau}}{h^{\alpha}} \sum_{k=-m+i}^{i} g_{k} u_{i-k}^{j}+\tau f_{i}^{j+\frac{1}{2}}
$$

For $i=1,2, \ldots, m-1, j=0,1, \ldots, N-1$,

$h=\frac{b-a}{m}, x_{i}=a+i h, \tau=\frac{T}{N}, t_{j}=j \tau$, and $u_{i}^{j}=u\left(x_{i}, t_{j}\right)$. We can rewrite the system (11) in matrix - vector form as

$$
U^{j+1}=(I-A) U^{j}+B C+\tau F^{j+\frac{1}{2}}
$$

where $U^{j}=\left(u_{1}^{j}, u_{2}^{j}, \ldots, u_{m-1}^{j}\right)^{T} F^{j+\frac{1}{2}}=\left(f_{1}^{j+\frac{1}{2}}, f_{2}^{j+\frac{1}{2}}, \ldots, f_{m-1}^{j+\frac{1}{2}}\right)^{T}$, $f_{i}^{j+\frac{1}{2}}=f\left(x_{i}, t_{j+\frac{1}{2}}\right), t_{j+\frac{1}{2}}=j \tau+\frac{\tau}{2}$ and $\mathrm{A}$ is an matrix $(\mathrm{m}-1) \times$ (m-1) with the entries $a=\frac{\tau D}{\left(h^{\alpha}\right)}, g_{k}=g_{-k}$, and BC is the vector
of boundary conditions.

$$
\begin{aligned}
A & =\left(\begin{array}{ccccc}
a g_{0} & a g_{-1} & a g_{-2} & \cdots & a g_{-m+2} \\
a g_{1} & a g_{0} & a g_{-1} & & a g_{-m+3} \\
a g_{2} & a g_{1} & a g_{0} & & a g_{-m+4} \\
\vdots & \vdots & & \ddots & \\
a g_{m-2} & a g_{m-3} & a g_{m-4} & & a g_{0}
\end{array}\right), \\
B C & =a\left(\begin{array}{c}
g_{1} u_{0}+g_{-m+1} u_{m} \\
g_{2} u_{0}+g_{-m+2} u_{m} \\
g_{3} u_{0}+g_{-m+3} u_{m} \\
\vdots \\
g_{m-1} u_{0}+g_{-1} u_{m}
\end{array}\right)
\end{aligned}
$$

\section{Stability of the Explicit Difference Approximation Method}

THEOREM 4.1 equation (12) for problem (2) and (3) is conditionally stable.

Proof. In [16] it was shown that the coefficients of the generating function (5) for $\mathrm{z} \in \mathrm{R}$ has the following form:

$|2 \sin (z / 2)|^{\alpha}=\sum_{k=-\infty}^{\infty} \frac{(-1)^{k} \Gamma(\alpha+1)}{\Gamma\left(\frac{\alpha}{2}-k+1\right) \Gamma\left(\frac{\alpha}{2}+k+1\right)} e^{i k h}$

Let $\lambda$ be the eigenvalue of matrix A, then by using (14) and Gerschgorin's circle theorem [19] we have:

$$
\left|\lambda-a g_{0}\right| \leq r_{i}=a \sum_{k=-m+i}^{i-1}\left|g_{k}\right|<a g_{0}
$$

Where $\sum_{k=-\infty}^{\infty}\left|g_{k}\right|=g_{0}$ that is, we have

$$
0<\lambda<2 a g_{0}
$$

For stability, the eigenvalues of matrix (I-A) should be satisfy $|1-\lambda|<1$, therefore, $0<\lambda<2$, Consequently $2 a g_{0}<2$, $a=\frac{\tau}{h^{\alpha}}<\frac{1}{g_{0}}$ and discrete equation (12) is conditionally stable.

\section{The Implicit Discretization for Fractional Diffusion Equation}

Stability of explicit methods was studied in the previous section. Now we want to discuss the implicit method. Implicit discretization for the equations 2 and 3 is

$$
\begin{aligned}
& \frac{u_{i}^{j+1}-u_{i}^{j}}{\tau}=-D h^{-\alpha} \sum_{k=-m+i}^{i} g_{k} u_{i-k}^{j+1}+f_{i}^{j+\frac{1}{2}} \\
& \text { Or } \\
& \qquad u_{i}^{j+1}+\frac{D \tau}{h^{\alpha}} \sum_{k=-m+i}^{i} g_{k} u_{i-k}^{j+1}=u_{i}^{j}+\tau f_{i}^{j+\frac{1}{2}}
\end{aligned}
$$

For $i=1,2, \ldots, m-1, j=0,1, \ldots, N-1$,

$h=\frac{b-a}{m}, x_{i}=a+i h, \tau=\frac{T}{N}, t_{j}=j \tau$, and $u_{i}^{j}=u\left(x_{i}, t_{j}\right)$. We can rewrite the system (18) in matrix - vector form as

$$
(I+A) U^{j+1}=U^{j}+B C+\tau F^{j+\frac{1}{2}}
$$

where $U^{j}=\left(u_{1}^{j}, u_{2}^{j}, \ldots, u_{m-1}^{j}\right)^{T}, F^{j+\frac{1}{2}}=\left(f_{1}^{j+\frac{1}{2}}, f_{2}^{j+\frac{1}{2}}, \ldots, f_{m-1}^{j+\frac{1}{2}}\right)^{T}$, $f_{i}^{j+\frac{1}{2}}=f\left(x_{i}, t_{j+\frac{1}{2}}\right), t_{j+\frac{1}{2}}=j \tau+\frac{\tau}{2}$, and $\mathrm{A}$ is an matrix $(\mathrm{m}-1) \times$ $(\mathrm{m}-1)$ with the entries $a=\frac{\tau D}{h^{\alpha}}, g_{k}=g_{-k}$, and BC is the vector of boundary conditions.

$A=\left(\begin{array}{ccccc}a g_{0} & a g_{-1} & a g_{-2} & \cdots & a g_{-m+2} \\ a g_{1} & a g_{0} & a g_{-1} & & a g_{-m+3} \\ a g_{2} & a g_{1} & a g_{0} & & a g_{-m+4} \\ \vdots & \vdots & & \ddots & \\ a g_{m-2} & a g_{m-3} & a g_{m-4} & & a g_{0}\end{array}\right)$,

$$
\mathrm{BC}=a\left(\begin{array}{c}
\left(g_{1} u_{0}^{j}+g_{-m+1} u_{m}^{j}\right)-\left(g_{1} u_{0}^{j+1}+g_{-m+1} u_{m}^{j+1}\right) \\
\left(g_{2} u_{0}^{j}+g_{-m+2} u_{m}^{j}\right)-\left(g_{2} u_{0}^{j+1}+g_{-m+2} u_{m}^{j+1}\right) \\
\left(g_{3} u_{0}^{j}+g_{-m+3} u_{m}^{j}\right)-\left(g_{3} u_{0}^{j+1}+g_{-m+3} u_{m}^{j+1}\right) \\
\vdots \\
\left(g_{m-1} u_{0}^{j}+g_{-1} u_{m}^{j}\right)-\left(g_{m-1} u_{0}^{j+1}+g_{-1} u_{m}^{j+1}\right)
\end{array}\right)
$$




\section{Stability of the Explicit Difference Approximation Method}

TheOREM 6.1 discrete equation (18) for problems (2) and (3) is unconditionally stable.

Proof: Let $\lambda$ is the eigenvalue of matrix $A$, then by using (14) and Gerschgorin's circle theorem [16] we have:

$$
\left|\lambda-a g_{0}\right| \leq r_{i}=a \sum_{k=-m+i}^{i-1}\left|g_{k}\right|<a g_{0}
$$

where $\sum_{k=-\infty}^{\infty}\left|g_{k}\right|=g_{0}$ that is, we have

$$
0<\lambda<2 a g_{0}
$$

Hence the eigenvalues of the matrix $(I+A)^{-1}$ satisfy $\frac{1}{|1+\lambda|}<1$ therefore, the spectral radius is less than one. Thus, the discrete equation (22) is unconditionally stable.

\section{Numerical Example}

For example, consider

$$
\begin{aligned}
& \frac{\partial u(x, t)}{\partial t}=D \frac{\partial^{\alpha} u(x, t)}{\partial|x|^{\alpha}}+f(x, t), \\
& a<x<b, \quad 0<t \leq T
\end{aligned}
$$

With the initial and boundary conditions

$\left\{\begin{array}{l}u(x, 0)=x^{2}(1-x)^{2}, \quad 0<x \leq 1 \\ u(0, t)=0, \quad 0<t \leq T \\ u(1, t)=0, \quad 0<t \leq T\end{array}\right.$

and with the non-homogeneous part

$f(x, t)=(1+t)^{-1+\alpha}(-1+x)^{2} x^{2} \alpha+$

$\frac{1}{\Gamma(5-\alpha)} x^{-\alpha}\left(\begin{array}{l}\left(\frac{1+t}{1-x}\right)^{\alpha}(-1+x)^{2} x^{\alpha} \\ \left(12 x^{2}-6 x \alpha+(-1+\alpha) \alpha\right)+ \\ (1+t)^{\alpha} x^{2}\left(\begin{array}{l}12(-1+x)^{2}+ \\ (-7+6 x) \alpha+\alpha^{2}\end{array}\right)\end{array}\right)$

$\sec \left(\frac{\pi \alpha}{2}\right)$

where the exact solution is

$$
u(x, t)=(t+1)^{\alpha} x^{2}(1-x)^{2}
$$

Figure 1 shows the solution profiles for the approximate solution computed using $a=1.2,1.4,1.8$ at $t=1$.

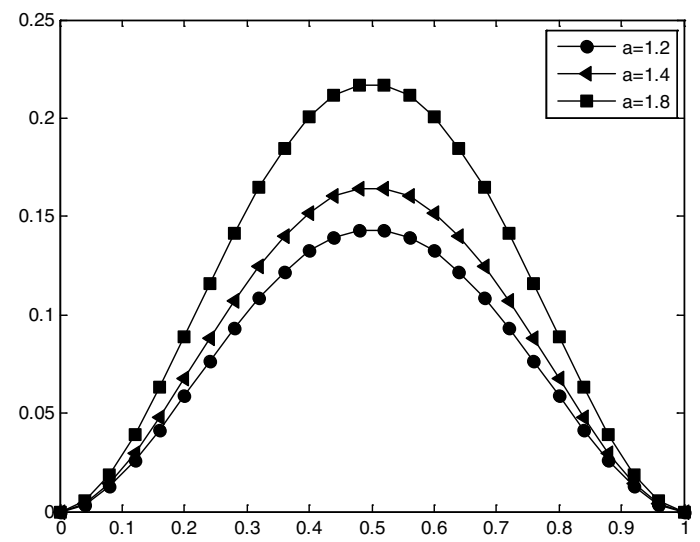

Figure 1. The exact solution of the Eqs. (23) with the initial and boundary conditions (24) at $t=1, a=1.2,1.4,1.8$.

Table 1. The maximum of errors for different values of $\alpha$ for $\tau=0.01, t=1$

\begin{tabular}{lcc}
\hline$\alpha$ & $\mathrm{h}=\mathbf{0 . 0 1}$ & $\mathrm{h}=\mathbf{0 . 0 0 5}$ \\
\hline 1.2 & $3.8857 \times 10^{-4}$ & $4.0142 \times 10^{-4}$ \\
1.4 & $5.2263 \times 10^{-4}$ & $5.4080 \times 10^{-4}$ \\
1.6 & $6.8416 \times 10^{-4}$ & $7.1023 \times 10^{-4}$ \\
1.8 & $8.7656 \times 10^{-4}$ & $9.1698 \times 10^{-4}$ \\
\hline
\end{tabular}

Table 2. The maximum of errors for different values of $\alpha$ for $\tau=0.01, t=5$

\begin{tabular}{cll}
\hline$\alpha$ & \multicolumn{1}{c}{$\mathrm{h}=\mathbf{0 . 0 1}$} & \multicolumn{1}{c}{$\mathrm{h}=\mathbf{0 . 0 0 5}$} \\
\hline 1.2 & $4.7954 \times 10^{-4}$ & $5.3553 \times 10^{-4}$ \\
1.4 & $7.8244 \times 10^{-4}$ & $8.7835 \times 10^{-4}$ \\
1.6 & $1.2 \times 10^{-3}$ & $1.4 \times 10^{-3}$ \\
1.8 & $1.9 \times 10^{-3}$ & $2.2 \times 10^{-3}$ \\
\hline
\end{tabular}

In Table 1, we calculated the maximum errors of the implicit method for several different values of $\alpha$ in interval $1<\alpha \leq 2$ at $t=1$ and $\tau=0.01$ when $h=0.01$ and $h=$ 0.005 .

In Table 2, we calculated the maximum errors of the implicit method for several different values of $\alpha$ in interval $1<\alpha \leq 2$ at $t=5$ and $\tau=0.01$, when $h=0.01$ and $\mathrm{h}=0.005$. Comparing the two tables shows that accuracy of this method in $t=1$ is more than in the time $\mathrm{t}=5$. When $\tau$ is considered as a constant, the accuracy of this method decrease in $\frac{h}{2}$.

In Table 3, we consider $h$ as a constant and obtain the maximum error of method for $\tau=0.01$ and $\tau=0.005$ in $x=0.4$. 
Table 3. The maximum of errors for different values of $\alpha$ for $h=0.01, x=0.4$

\begin{tabular}{ccc}
\hline$\alpha$ & $\tau=0.01$ & $\tau=0.005$ \\
\hline 1.2 & $3.5724 \times 10^{-4}$ & $1.7320 \times 10^{-4}$ \\
1.4 & $4.8062 \times 10^{-4}$ & $2.3164 \times 10^{-4}$ \\
1.6 & $6.2895 \times 10^{-4}$ & $3.0020 \times 10^{-4}$ \\
1.8 & $8.7656 \times 10^{-4}$ & $3.7824 \times 10^{-4}$ \\
\hline
\end{tabular}

Table 4. The maximum of errors for different values of $a$ for $h=0.01, x=0.7$

\begin{tabular}{ccc}
\hline$\alpha$ & $\tau=0.01$ & $\tau=0.005$ \\
\hline 1.2 & $2.7136 \times 10^{-4}$ & $1.3202 \times 10^{-4}$ \\
1.4 & $3.6542 \times 10^{-4}$ & $1.7638 \times 10^{-4}$ \\
1.6 & $4.7753 \times 10^{-4}$ & $2.2754 \times 10^{-4}$ \\
1.8 & $6.0919 \times 10^{-4}$ & $2.8423 \times 10^{-4}$ \\
\hline
\end{tabular}

In Table 4 we consider $h$ as a constant and obtain the maximum error of method for $\tau=0.01$ and $\tau=0.005$ in $x=0.7$. Comparing the two Tables 3,4 shows that accuracy of this method is increases by halving the $\tau$ and accuracy of this method increase when $x$ increase.

\section{Conclusions}

In this paper, Riesz derivative is approximated by using fractional central difference. Diffusion equation is approximated by Riesz derivative with applying the explicit and implicit numerical methods and we observed that after discretization for stability the spectral radius is less than one. The explicit method is conditionally stable and the stability of the implicit method is unconditional.

\section{Acknowledgement}

We would like to thank Saeed Rostami for many helpful suggestions.

\section{References}

1. Schneider W R, and Wyss W (1989). Fractional diffusion and wave equations, Journal of Mathematical Physics, vol 30(1), 134-144.
2. Wyss W (1986). The fractional diffusion equation, Journal of Mathematical Physics, vol 27(11), 2782-2785.

3. Sabatier J, Agrawal O P et al. (2007). Advances in Fractional Calculus: Theoretical Developments and Applications in Physics and Engineering, Springer, Netherlands, 175-182.

4. Magin R L (2006). Fractional Calculus in Bioengineering, Begell House Publisher., Inc., Connecticut, 721-732.

5. Scalas E, Gorenflo R et al. (2000). Fractional calculus and continuous - time finance, Physica A: Statistical Mechanics and its Applications, vol 284 (1-4), 376-384.

6. Gorenflo R, and Mainardi F (1998). Random walk models for space-fractional diffusion processes, Fractional Calculus and Applied Analysis, vol 1(2), 167-191.

7. Samko S G, Kilbas A A et al. (1993). Fractional integrals and derivatives: Theory and Applications, CRC Press, Gordon and Breach Science Publishers, 654-667.

8. Kilbas A A, Srivastava H M et al. (2006). Theory and Applications of Fractional Differential Equations, Elsevier Science Ltd, Amsterdam.

9. Podlubny I (1999). Fractional Differential Equations, Academic press, NewYork.

10. Tuan V K, and Gorenflo R (1995). Extrapolation to the limit for numerical fractional differentiation, Zeitschrift für angewandte Mathematik und Mechanik, vol 75(8), 646-648.

11. Meerschaert M M, and Tadjeran C(2006). Finite difference approximations for two - sidedspace-fractional partial differential equations, Applied Numerical Mathematics, vol 56(1), 80-90.

12. Tadjeran C, Meerschaert M M et al. (2006). Asecond-order accurate numerical approximation for the fractional diffusion equation, Journal of Computational Physics, vol 213(1), 205-213.

13. Shen S, Liu F (2011). Numerical approximations and solution techniques for the space - time Riesz-caputo fractional advection-diffusion equation, Numerical Algorithms, vol 56(3), 383-403.

14. Zhuang P, Liu F et al. (2009). Numerical methods for the variable-order fractional advection-diffusion equation with a nonlinear source term, SIAM Journal on Numerical Analysis, vol 47(3), 1760-1781.

15. Zhang H, Liu F (2008). Numerical simulation of the Riesz fractional diffusion equation with a nonlinear source term, Journal of Applied Mathematics and Informatics, vol 26(1-2), 1-14.

16. Ortigueira M D (2006). Riesz potential operators and inverses via fractional centred derivatives, International Jour-nal of Mathematics and Mathematical Sciences, vol 2006, 1-12.

17. Kincaid D, and Cheney W (1991). Numerical Analysis, Brooks/Cole Pub., California. 